\title{
Discussion - Circumstellar environment of active OB stars
}

\author{
summarized by Douglas R. Gies ${ }^{1}$ and Richard H. D. Townsend ${ }^{2}$ \\ ${ }^{1}$ Center for High Angular Resolution Astronomy and Department of Physics and Astronomy, \\ Georgia State University, P. O. Box 4106, Atlanta, GA 30302-4106, U.S.A. \\ ${ }^{2}$ Department of Astronomy, University of Wisconsin-Madison, Madison, WI 53706, USA
}

This session dealt with the circumstellar gas surrounding active OB stars, and the discussion broadly focused on disks, wind morphology and structures, X-ray emission, and mass loss rates.

Alex Lobel noted that the GAIA mission will record the Paschen $14 \lambda 8598$ line of hydrogen, and he asked about the state of models of line formation in Be star disks with cooler, equatorial zones. Alex Carciofi responded that two-dimensional models for optically thick disks solved for the radiation field and temperature distribution selfconsistently. He suggested that even high level transitions of hydrogen, like Paschen 14, were probably optically thick out to regions well above and below the cooler equatorial zone in Be stars with dense disks. Vladimir Strelnitski called attention to MWC349, an object hosting a disk with possible solid-body instead of Keplerian rotation.

Thomas Rivinius commented on the claims from interferometric observations of polar winds in Be stars. He doubted that significantly dense polar winds exist because: (1) the winds from the polar regions of Be stars should be similar to those of normal B-stars, i.e., relatively weak; (2) if optically thick polar winds are present, they should appear in other kinds of observations (e.g., emission lines); (3) absolute visibility measurements with VLTI/Amber are difficult, and the detection of polar winds from other interferometers (NPOI, CHARA) has not been forthcoming. Jo Puls added that wind models indicate that the IR continuum emission might extend to 1.2 stellar radii but not to 10 stellar radii. Philippe Stee responded that while the interferometric results are interpreted using a polar wind model, the emission may result instead from a disk wind. Anthony Meilland commented that some image reconstruction work on VLTI/VINCI observations of the Be star Achernar yields evidence of polar winds without relying on a wind model framework.

The discussion then turned to the issue of the hour-glass shaped outflows from LBVs and other luminous stars. Stan Owocki noted that the Homunculus Nebula surrounding $\eta$ Carinae probably resulted from faster wind flows and higher mass loss from the polar regions. He asked if there is any evidence that more mass is ejected from the polar regions of LBVs in general. Kerstin Weis responded that many LBVs show bipolar outflows, but the mass loss rates as a function of latitude were not well known. Nathan Smith added that the answer requires data on both gas density and solid angle of emission, and such work has only been done for $\eta$ Carinae and P Cygni. Dietrich Baade pointed out that a similar hour-glass morphology exists around the site of SN1987A (whose progenitor was a blue-supergiant). He advocated a search for circumstellar gas envelopes around other early-type supergiants, especially in the Magellanic Clouds where the extinction is low. He wondered if there was a consensus about the origin of bipolar outflows in rotation and/or magnetic fields. Baade also reminded us that supernovae often show evidence of asymmetries (detected through polarimetry), and he speculated about a general connection between the asymmetries pre- and post-SN. Nathan Smith responded 
that in the case of SN1987A, high resolution observations of the expanding cloud from the explosion displayed an asymmetry orthogonal to that of the shells from the pre-SN wind loss, so he doubted that the SN asymmetries were related to the pre-SN rotation axis.

Coralie Neiner redirected the discussion to X-rays from active OB stars. She noted that many of the newly detected magnetic OB stars from the MiMeS program belong to the weak-wind category, and she asked about the X-ray properties of the stars with measured magnetic fields. David Cohen responded that there is a large diversity of X-ray properties among the magnetic stars, ranging from strong X-ray emitters like $\theta^{1}$ Ori $\mathrm{C}$ to rather weakly X-ray emitting stars.

Nathan Smith brought up the all-important issue of mass loss rates. He noted that the mass loss rates derived from X-ray studies are lower than those from standard UV line analyses and are closer to those for clumpy winds. He asked whether X-ray diagnostics are based upon the wind properties in environments with similar densities to those for the UV line studies. David Cohen answered affirmatively that the X-rays form throughout the wind and act to back-light the wind material seen along the line of sight. He noted that the K-shell edges seen in Chandra X-ray spectroscopy of O-stars arise from similar ions to those that form $\mathrm{P}$ Cygni wind lines in the UV spectrum.

Rich Townsend raised the issue of porosity in the wind, i.e., the idea that the wind is spatially porous and structured into optically thick clumps (see Owocki \& Cohen 2006, ApJ, 648, 565). David Cohen emphasized that creating such optically thick clumps would require sweeping up wind gas over a large length scale. Rayleigh-Taylor instabilities would likely develop and make smaller clumps. Thus, if porosity is invoked to revise upwards mass loss rates from X-ray diagnostics, then clumping must inevitably follow, and hence mass loss rate indicators based upon density squared diagnostics would need to be lowered. Jorick Vink discussed the similarity of the mass loss rates from theory with those from $\mathrm{H} \alpha$ that include the downward revision from clumping, while for wind models invoking porosity, the theoretical and observationally implied mass loss rates differ. David Cohen concluded the discussion with a plea for more research on the mass loss rates and all their observational diagnostics (X-ray, $\mathrm{H} \alpha, \mathrm{P}$ V lines, IR and radio continuum excess, etc.). He noted the development of a new code by Rich Townsend that characterizes the wind clumping properties and derives estimates of observable quantities like the net polarization. 